\title{
Extracapsular Cataract Extraction and Posterior Chamber Lens Implantation in Patients with Primary Chronic Angle-Closure Glaucoma: Effect on Intraocular Pressure Control
}

\author{
P. K. WISHART, P. L. ATKINSON \\ Liverpool
}

\begin{abstract}
Summary
Twenty-three eyes of 22 consecutive patients with primary chronic angle-closure glaucoma (PCACG) undergoing extracapsular cataract extraction (ECCE) with intraocular lens (IOL) implantation were studied prospectively to assess the effect of ECCE on intraocular pressure (IOP) control. For comparison, a group of 21 eyes with open angle glaucoma (OAG) which had undergone ECCE were reviewed retrospectively. The PCACG group had a low incidence of acute post-operative IOP elevations $(9 \%)$, and long-term the majority $(65 \%)$ achieved an IOP $<21 \mathrm{mmHg}$ off all glaucoma medications. In the OAG group the IOP control was largely unaffected. We recommend that eyes with cataract and PCACG should be considered for ECCE as a first procedure rather than combined cataract and filtering surgery.
\end{abstract}

The optimal management of coexisting cataract and glaucoma remains undecided. The surgical options are to achieve IOP control by filtration surgery as a separate procedure, or to perform a cataract extraction combined with filtering surgery. Studies on patients with open angle glaucoma (OAG) have shown that long-term intraocular pressure (IOP) control is largely unaffected by cataract surgery alone, ${ }^{1-3}$ although acute post-operative IOP rises following extracapsular cataract extraction (ECCE) with intraocular lens (IOL) implantation is a well documented finding. ${ }^{2-5}$

In our experience, patients with primary chronic angle-closure glaucoma (PCACG) seldom show an acute elevation of IOP following ECCE with IOL implantation. This prospective study, therefore, was undertaken to determine the effect of ECCE with IOL implantation on IOP control in patients with
PCACG and cataract. To serve as a comparison a retrospectively selected group of patients with OAG who had undergone extracapsular cataract surgery was also studied.

\section{Materials and methods}

Twenty-four eyes of 23 consecutive patients with PCACG and visually significant cataracts undergoing ECCE with IOL implantation were followed prospectively. All cases included were of primary angle-closure glaucoma in which cataract had developed several years after iridectomy/iridotomy. In no case was the glaucoma secondary to iritis or disease of the lens such as phakolytic glaucoma or lens subluxation. Twelve eyes had had a previous episode of acute angle-closure glaucoma, seven eyes had presented as subacute angle-closure, and five eyes had presented as chronic angle-closure glaucoma 
with a shallow anterior chamber and peripheral anterior synechiae (PAS) of the anterior chamber angle demonstrated by Zeiss four mirror indentation gonioscopy. All eyes had previously had a peripheral iridectomy (20 eyes) or laser iridotomy (four eyes) performed and all were receiving topical and in some cases systemic anti-glaucoma medications.

All patients underwent initial slit-lamp eye examination including Goldmann applanation tonometry, anterior chamber depth measurement using a Haag-Streit pachymeter, gonioscopy using a Zeiss four mirror indentation gonioscope by one author (PKW) to assess the extent of peripheral anterior synechiae (PAS), and where possible fundoscopy and disc assessment. Pre-operative IOP was taken as the mean of the last three recorded measurements prior to surgery.

Eyes with long-standing PCACG and cataract in which pre-operative indentation gonioscopy showed near total synechial closure of the anterior chamber angle with PAS extending to Schwalbe's line, and medically uncontrollable IOP, were excluded from the study as it was felt that for these eyes ECCE combined with trabeculectomy was indicated.

Extracapsular cataract extraction with implantation of a posterior chamber IOL was performed in all cases, under general anaesthetic in all but one case. A corneal section was employed in all cases. Posterior synechiae were broken down using Healonid and an iris spatula, and sphincterotomies performed where necessary. Healonid was used in all cases and was washed out at the end of each operation. The section was closed with $10 / 0$ nylon either as a continuous or interrupted sutures.

Post-operatively, IOP was recorded for the first two days and if satisfactory the patient discharged home off glaucoma treatment and using topical dexamethasone or betamethasone four times a day and a mydriatic. Patients were followed up regularly in the outpatient clinic and glaucoma therapy recommenced if felt clinically necessary.

At the end of the study period all patients had repeat indentation gonioscopy (PKW) and optic disc assessment by slit-lamp biomicroscopy.
For comparison, the notes of 20 patients (21 eyes) with chronic simple glaucoma (CSG) who had undergone extracapsular cataract surgery were reviewed retrospectively. Nineteen eyes were diagnosed as suffering from OAG while we classified two eyes as suffering from 'narrow angle' glaucoma (NAG). These latter two eyes had shallow anterior chambers $(<2.5 \mathrm{~mm})$ but no PAS as demonstrated by Zeiss four mirror indentation gonioscopy. Except in one eye with OAG who had had a failed trabeculectomy, no patient had undergone previous surgery or laser treatment, and all had normal IOPs controlled with medical treatment. A posterior chamber IOL was implanted in 17 cases, the remaining four patients receiving no IOL.

\section{Results}

\section{PCACG Group}

Of the 23 patients in the CACG group, 22 patients (23 eyes) completed follow up. One patient, who had originally presented with subacute angle-closure glaucoma, suffered a central retinal vein occlusion two weeks postoperatively and developed rubeotic glaucoma. This patient is excluded from the following analysis.

The CACG group consisted of nine male and 13 female patients, mean age 75.4 years (standard deviation SD $=6.7$ ), range 62-86 years.

Mean anterior chamber depth was $1.85 \mathrm{~mm}$ $(\mathrm{SD}=0.24)$, range $1.3-2.05 \mathrm{~mm}$.

Mean post-operative follow up was 11.2 months $(\mathrm{SD}=6.7)$, range 6-24 months.

Mean pre-operative IOP was $19.1 \mathrm{mmHg}$ $(\mathrm{SD}=3.1)$, range $13-25 \mathrm{mmHg}$.

Post-operatively, at the end of the study period, the mean IOP was $15.6 \mathrm{mmHg}$ $(\mathrm{SD}=3.3)$, range $12-25 \mathrm{mmHg}$.

In 15 eyes the IOP was controlled $(<21 \mathrm{mmHg})$ off all medications.

IOP was controlled on fewer medications in $18 / 23$ eyes; in 15 of those eyes the IOP was lower than pre-operatively.

Control of IOP was unchanged on the same medications in 3 of the 23 eyes.

Two eyes required more medications postoperatively than pre-operatively. One of these eyes required an increase in the strength of pilocarpine drops and the other the addi- 
tion of $125 \mathrm{mg}$ per day acetazolamide. In this latter case the post-operative IOP was $20 \mathrm{mmHg}$ on the original medications, the extra medication being added in an effort to reduce the IOP still further in an eye with severe glaucomatous damage. Table I shows the number of glaucoma medications in use pre- and post-operatively.

All patients in the PCACG group had a degree of glaucomatous optic disc damage. Of the two patients who required more medications post-operatively both had end-stage glaucomatous disc cupping; however, of the five patients with end-stage cupping three patients had improved control post-operatively.

In the first 48 hours post-operatively two patients were recorded as having a significant IOP rise $(>6 \mathrm{mmHg}$ over pre-operative IOP)—having pressures of $40 \mathrm{mmHg}$ on day one and $30 \mathrm{mmHG}$ on day two respectively.

A post-operative IOP rise which was considered to be a steroid response was seen in 2 of the 23 patients, the IOP normalising on cessation of the steroids.

One patient from this group developed cystoid macular oedema post-operatively, reducing the final visual acuity to 6/18. Another patient developed severe anterior uveitis six weeks post-operatively which responded to treatment with topical steroids.

The glaucomatous damage to the optic discs was classifed into three groups: 'Good', 'moderately damaged', and 'bad'. In the PCACG patients the discs were assessed as five good, eight moderate, and ten badly damaged.

\section{CSG Group}

This group consisted of 21 eyes of 20 patients,

Table I Preoperative versus Postoperative medications for the PCACG group

\begin{tabular}{|c|c|c|}
\hline $\begin{array}{l}\text { PCACG Group } \\
\text { No. of } \\
\text { Medications }\end{array}$ & Number (\%) & Eyes $(N=23)$ \\
\hline Medications & Preoperative & Postoperative \\
\hline 0 & 0 & $15(65)$ \\
\hline 1 & $11(48)$ & $4(17)$ \\
\hline 2 & 10 (44) & $3(13)$ \\
\hline 3 & $2(9)$ & $1(4)$ \\
\hline $\begin{array}{l}\text { Mean No. } \\
\text { Medications }\end{array}$ & 1.61 & 0.57 \\
\hline
\end{tabular}

five male and 15 female. Mean age was 77.6 years $(S D=11.3)$, range $55-93$ years.

Mean post-operative follow up was 14.8 months $(S D=11.5)$, range 6-48 months.

Mean pre-operative IOP was $19.4 \mathrm{mmHg}$ $(\mathrm{SD}=3.2)$, range $14-28 \mathrm{mmHg}$.

Mean post-operative IOP at the end of the follow up period was $19.6 \mathrm{mmHg}(\mathrm{SD}=3.8)$, range $14-28 \mathrm{mmHg}$.

In one eye the IOP was controlled $(<21 \mathrm{mmHg})$ off all medications.

IOP was controlled on fewer medications in four eyes; in all of these cases the IOP was lower than the pre-operative level.

In 12 eyes the glaucoma medication remained unchanged.

Five eyes required more glaucoma medication post-operatively. Of these eyes, four required their medication increased from one to two topical drops. The remaining case required insertion of a Molteno drainage tube five months post-operatively in order to control the IOP.

Table II shows the number of glaucoma medications in use pre- and post-operatively.

In the first 48 hours post-operatively three eyes were recorded as having a signifiant IOP rise-having IOPs of 30 and $32 \mathrm{mmHg}$ on day one and $29 \mathrm{mmHg}$ on day two respectively.

Three eyes experienced a rise in IOP in the post-operative period which was attributed to a steroid response.

Of the two eyes considered to have narrow angle glaucoma, control in one eye was unchanged and in the other improved (controlled on fewer medications). In the CSG group the optic discs were assessed as six good, ten moderately damaged, and five badly damaged.

One eye required an anterior vitrectomy following vitreous loss per-operatively and did not receive an IOL. This patient had unchanged control post-operatively. In another patient large choroidal effusions ocurred post-operatively which resolved spontaneously.

Table III summarises the differences in IOP control between the two groups.

Change in anterior chamber angle after $E C C E+I O L$

In the CSG group the anterior chamber angles 
Table II Preoperative versus Postoperative medications for the CSG group

\section{CSG Group}

No. of

Medications

Number $(\%)$ of Eyes $(N=21)$

Preoperative Postoperative

\begin{tabular}{ccr}
\hline 0 & 0 & $1(5)$ \\
1 & $14(67)$ & $11(52)$ \\
2 & $5(24)$ & $6(29)$ \\
3 & $1(5)$ & $1(5)$ \\
4 & 0 & $1(5)$ \\
\hline
\end{tabular}

Mean No.

Medications

1.29

1.43

were open pre-and post-operatively, with no forward bowing of the iris. Post-operatively, there was some increase in peripheral anterior chamber depth as assessed with the slit-lamp, and the anterior chamber angle remained open.

In the PCACG group, in 22 of the 23 eyes, there was a marked flattening of the forward convexity of the peripheral iris and deepening of the anterior chamber angle post-operatively.

Table IV summarises the relationships between post-operative medications, PAS, and pre-operative anterior chamber depth.

Despite patent peripheral iridectomies/ laser iridotomies in all eyes, pre-operative gonioscopy was difficult in some cases due to extreme narrowness of the anterior chamber angle. Accurate assessment was impossible in eight eyes, but seven eyes were judged to have no PAS pre-operatively, two eyes to have limited PAS, and six eyes to have extensive PAS.

Post-operative assessment at six months follow-up was much easier in that the peripheral anterior chamber deepened considerably

Table III Comparison of IOP control between the $P C A C G$ and $C S G$ groups

\begin{tabular}{lrr}
\hline & \multicolumn{2}{c}{ No. of Patients (\%) } \\
& $\begin{array}{l}\text { PCACG } \\
(N=23)\end{array}$ & $\begin{array}{c}C S G \\
(N=21)\end{array}$ \\
\hline Early IOP rise & $2(9)$ & $3(14)$ \\
Steroid response & $2(9)$ & $1(5)$ \\
IOP <21mmHg: & $15(65)$ & $1(5)$ \\
Off all medications & $3(13)$ & $4(19)$ \\
Fewer medications & $3(13)$ & $12(57)$ \\
Same medications & $2(9)$ & $5(23)$ \\
More medications & &
\end{tabular}

in 22 of the 23 eyes. In the 15 eyes assessed for PAS pre-operatively there was good concordance with the post-operative assessment in 12 eyes especially where the angle was judged to be extensively closed pre-operatively. No PAS closure of the angle was confirmed in six of the seven eyes judged to be open pre-operatively, but one eye (which required an increase in medication post-operatively) was found to have suffered 300 degree closure (No.22). The only anterior chamber to remain extremely shallow post-operatively also had extensive PAS closure (No.23).

\section{Discussion}

The PCACG and CSG groups are not directly comparable, firstly because of the different sampling techniques and secondly because of the severity of the glaucoma. The PCACG group were using a greater mean number of medications preoperatively and also showed more optic disc damage. In addition, due to

Table IV Post-operative medications, PAS, and preoperative anterior chamber depth in the PCACG group.

\begin{tabular}{|c|c|c|c|}
\hline $\begin{array}{l}\text { Post-op. } \\
\text { medications }\end{array}$ & Eye & $\begin{array}{c}P A S \text { (degrees) } \\
\text { assessed post-op. }\end{array}$ & $\begin{array}{c}A C D(\mathrm{~mm}) \\
\text { (pre-op.) }\end{array}$ \\
\hline Nil & 1 & Nil & 1.3 \\
\hline$"$ & 2 & $"$ & 2.37 \\
\hline$"$ & 3 & $"$ & 2.0 \\
\hline$"$ & 4 & $"$ & 2.0 \\
\hline$"$ & 5 & $"$ & 1.85 \\
\hline$"$ & 6 & $"$ & 1.73 \\
\hline$"$ & 7 & $"$ & 2.0 \\
\hline$"$ & 8 & $"$ & 2.3 \\
\hline$"$ & 9 & 40 & 1.85 \\
\hline$"$ & 10 & 50 & 2.1 \\
\hline$"$ & 11 & 270 & 1.7 \\
\hline$"$ & 12 & 360 & 2.0 \\
\hline$"$ & 13 & 300 & 2.0 \\
\hline$"$ & 14 & 180 & 1.5 \\
\hline$"$ & 15 & 90 & 2.0 \\
\hline Fewer & 16 & Nil & 1.8 \\
\hline$"$ & 17 & 90 & 1.7 \\
\hline$"$ & 18 & 90 & 1.9 \\
\hline Same & 19 & Nil & 1.8 \\
\hline$"$ & 20 & Nil & 1.9 \\
\hline$"$ & 21 & 360 & 2.0 \\
\hline More & 22 & 300 & 2.0 \\
\hline$"$ & 23 & 260 & 1.9 \\
\hline
\end{tabular}

$(\mathrm{ACD}=$ anterior chamber depth) 
the previous surgical iridectomies, the cataract surgery in the PCACG group was generally more complicated, resulting in more intraocular manipulation and pigment release.

Notwithstanding the above considerations, the PCACG group had a low incidence of early post-operative IOP rises $(9 \%)$. This compares to $14 \%$ in the CSG group and is low compared with other studies on patients with CSG. $^{2-4}$

The incidence of post-operative steroid responders was $9 \%$ and $5 \%$ in the PCACG and CSG groups respectively. This is as expected from the reported high incidence of steroid response in both $\mathrm{CSG}^{6,7}$ and angle closure glaucoma. ${ }^{8}$

In the long-term, ECCE with IOL implantation had a beneficial effect on the IOP control of patients in the PCACG group, with a mean IOP reduction of $3.5 \mathrm{mmHg}(\mathrm{p}<0.01)$ on 1.04 mean fewer medications $(\mathrm{p}<0.001)$. In this group the majority $(65 \%)$ had a longterm IOP less than $21 \mathrm{mmHg}$ on no medication, although some eyes with severe glaucomatous damage were prescribed glaucoma medications in an attempt to reduce the IOP still further. In the CSG group the largely unchanged control is in keeping with previous studies. ${ }^{1-3}$

Our results agree with those of Greve ${ }^{9}$ who found that lens extraction was beneficial in terms of IOP control in patients with angle closure glaucoma. In Greve's study the extent of pre-operative PAS did not influence the post-operative IOP control.

In our study, the degree of pre-operative PAS also appeared to have little effect on long-term IOP control in the PCACG group. Two eyes in this group had worse post-operative IOP control, and these eyes had extensive closure of the anterior chamber angle with PAS. It might be reasonable to attribute this failure of IOP control to PAS closure of the angle, but three other eyes in this study had as extensive PAS and their glaucoma control improved post-operatively. Within the range of 1.3 to $2.3 \mathrm{~mm}$, the shallowness of the anterior chamber was not related to the degree of PAS or change in IOP control (Table IV).

Two factors may be responsible for the raised IOP secondary to an attack of acute or subacute angle-closure glaucoma: (1) mechanical closure of the angle by PAS, and (2) damage to the trabecular meshwork secondary to the high IOP and inflammation associated with an attack. Therefore, the degree of PAS closure visible on indentation gonioscopy may not reflect the true extent of trabecular meshwork damage sustained in the acute attack. Furthermore, there are also variations in the antero-posterior extent of the trabecular meshwork involved in PAS, and it may be that very anteriorly extending PAS are more significant than those with only a short anterior extent.

Perhaps a third factor responsible for elevated IOP in the light of our results is some mechanical impediment to aqueous outflow secondary to a large cataractous lens.

Few previous reports have investigated the effect on IOP control of cataract surgery in patients with PCACG, other studies dealing mainly with patients with OAG or glaucoma of mixed types. Simmons et al. ${ }^{10}$ retrospectively reviewed 75 glaucoma patients, including 15 eyes with PCACG, undergoing combined cataract and filtration surgery and found improved IOP control post-operatively but did not present the results for the PCACG patients separately. In their study $33 \%$ of patients had an early post-operative IOP rise of greater than $7 \mathrm{mmHg}$ despite combined surgery, compared to reported incidences of up to $72 \%$ in patients with glaucoma undergoing cataract surgery alone. ${ }^{2}$ Savage et al. ${ }^{2}$ reviewed a series of patients with glaucoma undergoing cataract surgery which included 12 eyes which had had an episode of acute angle-closure but had normal IOP pre-operatively. They found that the incidence of early IOP rises in this group approximated to that seen in non-glaucomatous eyes.

Modern ECCE with posterior chamber IOL implantation is a relatively safe and effective technique for the removal of visually handicapping cataracts in patients with glaucoma. Because of the danger of an acute postoperative rise in $\mathrm{IOP}^{2-5}$ and the necessity for long-life anti-glaucoma medications some authors recommend ECCE and IOL combined with filtering surgery., ${ }^{4,10}$ Combined surgery, however, must put the eye at greater 
risk of per-operative complications and Simmons et al. ${ }^{10}$ report only a $50 \%$ long-term control of glaucoma off medications with this procedure.

In patients with PCACG, either following an acute or subacute attack, or with creeping angle closure, assessment of the degree of glaucomatous damage may be difficult. Following a surgical peripheral iridectomy/laser iridotomy and long term miotic treatment optic disc assessment and visual field analysis is hindered by miosis, posterior synechiae of the pupil, and coexisting cataract. In eyes with PCACG, if cataract extraction is performed without simultaneous filtration surgery there might be expected to be a risk of IOP elevation in the post-operative period, causing considerable damage to an already compromised optic disc. ${ }^{14}$

The results of our study indicate that ECCE with IOL implantation alone will benefit the IOP control in the majority of patients with PCACG. The reason for this effect is unclear. Removing a cataract in an eye with OAG would be unlikely to affect long-term glaucoma control and most studies reflect this view. ${ }^{1-3}$ In our study, eyes with OAG undergoing ECCE with IOL implantation alone did not have improved IOP control post-operatively. Yet there was a clear improvement in IOP control in the majority of patients with PCACG undergoing ECCE with IOL implantation, with $65 \%$ of eyes requiring no medications post-operatively. There was also an unexpectedly low incidence of early postoperative IOP rises considering the extent of glaucomatous damage most of these eyes had suffered.

Pupil block had previously been relieved in these eyes and therefore this could not be a factor in the improved post-operative IOP control. It may be postulated that a large cataractous lens in an eye with a relatively small anterior segment somehow adversely affects the outflow of aqueous from the eye. In the PCACG group, the low incidence of early post-operative IOP elevations suggests an immediate beneficial effect on aqueous outflow. In the majority of cases the anterior chamber angle widened and the peripheral anterior chamber deepened post-operatively, and it may be that this change in the config- uration of the anterior chamber angle was responsible for an improvement in the function of the trabecular meshwork, perhaps by relieving physical compression of the trabecular lamellae. However, other factors such as an increase in uveoscleral outflow may be implicated.

The beneficial effect of ECCE and IOL implantation in these eyes with PCACG raises the question, should this procedure be considered in eyes with PCACG, uncontrolled IOP, and a clear lens? Greve certainly believes this to be the case as filtering surgery in such eyes may subsequently cause the lens to opacify, and he believes that lens extraction removes the underlying cause of the condition-namely a large lens. ${ }^{9}$

Might, therefore, ECCE and IOL implantation be beneficial to eyes with uncontrolled IOP and narrow angle glaucoma? A study of argon laser trabeculoplasy (ALT) in eyes with narrow angle glaucoma showed that following relief of pupil block the anterior chamber angle widened sufficiently to permit ALT in the majority of eyes. ${ }^{15}$ However, combined peripheral iridectomy/laser iridotomy plus ALT failed to improve IOP control in $50 \%$ of eyes treated. It should be noted, however, that 18 of the 23 eyes $(78 \%)$ in our PCACG group had previously suffered an attack of acute or subacute angle-closure glaucoma and the other five had chronic angle-closure glaucoma with significant closure of the anterior chamber angle by PAS. In the NAG study ${ }^{15}$ the majority of eyes were hypertensive eyes with narrow angles and those with PAS closure were of the insidious creeping closure variety. It is therefore likely that many eyes in the NAG group were suffering from chronic simple glaucoma coincident with a narrow anterior chamber angle and some degree of appositional closure leading to PAS. This may explain why peripheral iridectomy/laser iridotomy alone did not improve significantly IOP control, as pupil block was not contributing to the raised IOP. Re-analysis of the results of this NAG study presented at the 3rd Congress of the European Glaucoma Society, showed a close correlation between failure of IOP control and extension of PAS, ${ }^{16}$ probably related to the ALT. In our study, we found no improvement in IOP control in eyes with CSG 
following ECCE and IOL implantation, and therefore as CSG may be the underlying mechanism for the IOP elevation in many cases of NAG the authors would not recommend lens extraction as a therapeutic procedure in NAG eyes.

\section{Conclusion}

In eyes with cataract and primary chronic angle-closure glaucoma under reasonable medical control we recommend ECCE with IOL implantation as a first procedure rather than cataract extraction combined with filtering surgery.

We are indebted to Mrs C. Owen for her administrative and technical help.

\section{References}

${ }^{1}$ Radius RL, Schultz K, Sobocinski K, Schultz RO, Eason H: Pseudophakia and Intraocular Pressure. Am J Ophthalmol 1984, 97: 738-42.

${ }^{2}$ Savage JA, Thomas JV, Belcher CD, Simmons RJ: Extracapsular cataract extraction and posterior chamber intraocular lens implantation in glaucomatous eyes. Ophthalmology 1985, 92: 1506-16.

${ }^{3}$ McGuigan LJB, Gottsch J, Stark WJ, Maumenee AE, Quigley HA: Extracapsular cataract extraction and posterior chamber lens implantation in eyes with preexisting glaucoma. Arch Ophthalmol 1986, 104: 1301-8.

${ }^{4}$ Feitl ME, Krupin T, Bishop KI: Postoperative intraocular pressure following cataract or combined cataract-filtering surgery in open angle glaucoma. Presented at the 3rd Congress of the European Glaucoma Society, Estoril, May 1988.

${ }^{5}$ Gross JG, Meyer DR, Robin AL, Filar AA, Kelley JS: Increased intraocular pressure in the immediate postoperative period after cataract extraction. Am J Ophthalmol 1988, 105: 466-9.
${ }^{6}$ Becker B and Mills DW: Corticosteriods and intraocular pressure. Arch Ophthalmol 1963, 70: 500-7.

${ }^{7}$ Becker B and Hahn KA: Topical corticosteroids and heredity in Open Angle Glaucoma. Am J Ophthalmol 1964, 57: 543-51.

${ }^{8}$ Akingbehin AO: Corticosteroid induced Ocular Hypertension. I. Prevalence in Closed Angle Glaucoma. Br J Ophthalmol 1982, 66: 536-40.

${ }^{9}$ Greve EL: Primary Angle Closure Glaucoma: Extracapsular Cataract Extraction or Filtering Procedure? International Ophthalmology 1988, 12: $157-62$.

${ }^{10}$ Simmons ST, Litoff D, Nichols DA, Sherwood MB, Spaeth GL: Extracapsular Cataract Extraction and Posterior Chamber Intraocular Lens Implantation Combined with Trabeculectomy in Patients with Glaucoma. Am J Ophthalmol 1987, 104: 465-70.

${ }^{11}$ McCartney DL, Memmon JE, Stark WJ, Quigley HA, Maumenee AE, Gottsch JD, Bernitsky DA, Wong SK: The Efficacy and Safety of Combined Trabeculectomy, Cataract Extraction, and Intraocular Lens Implantation. Ophthalmology 1988, 95: 754-63.

${ }^{12}$ Percival SPB: Glaucoma Triple Procedure of Extracapsular Cataract Extraction, Posterior Chamber Lens Implantation, and Trabeculectomy. $\mathrm{Br} J$ Ophthalmol 1985, 69: 99-102.

${ }^{13}$ Jay JL: Extracapsular Lens Extraction and Posterior Chamber Intraocular Lens Insertion combined with Trabeculectomy. Br J Ophthalmol 1985, 69: 487-90.

${ }^{14}$ Hayreh SS: Anterior Ischaemic Optic Neuropathy IV. Occurrence after Cataract Extraction. Arch Ophthalmol 1980, 98: 1410-16.

${ }^{15}$ Wishart PK, Nagasubramanian S, Hitchings RA: Argon laser trabeculoplasty in Narrow Angle Glaucoma. Eye 1987, 1: 567-76.

${ }^{16}$ Wishart PK, Nagasubramanian S, Hitchings RA: Argon laser trabeculoplasty in Narrow Angle Glaucoma. Presented at the 3rd Congress of the European Glaucoma Society, Estoril, May 1988. 